\title{
OPERANT MODEL OF FRUSTRATED EXPECTED REWARD IN MICE
}

Aurelijus Burokas ${ }^{\mathrm{a}^{*}}$, Javier Gutiérrez-Cuesta ${ }^{\mathrm{a}^{*}}$, Elena Martín-García ${ }^{\mathrm{a}^{*}}$ and Rafael Maldonado ${ }^{\mathrm{a}}$

${ }^{a}$ Departament de Ciencies Experimentals i de la Salut, Universitat Pompeu Fabra, PRBB, C/ Dr. Aiguader 88, 08003, Barcelona, Spain

Correspondence should be addressed to Rafael Maldonado: rafael.maldonado@upf.edu Phone: +34-93-3160824

Fax: +34-93-3160901

Category of the article: Regular research article

Number of references: 30

Words in Abstract: 163

Words in Article (excluding abstract, references and figure legends): 5606

Number of figures: 8

Number of tables: 0

Supplemental information: 1

Short title: Model of frustration in mice

* These authors contributed equally to this work 


\section{Abstract}

One aspect of the addictive process that has not been thoroughly investigated is the consequence of the frustrated state occurring when the drug is not available. The present study aimed to validate a novel operant model of frustrated expected reward in mice. C57BL/6J mice were trained in operant conditioning maintained by chocolate-flavoured pellets or cocaine. After the completion of high rates of responding on a progressive ratio schedule, the reward was unexpectedly withheld. The consequences of this frustrated behaviour on anxiety, aggressiveness, perseveration, extinction and reinstatement were investigated. Mice exposed to the frustrated event perseverated in the operant responses and showed increased aggressiveness in the resident-intruder test. These animals also showed higher rates of cue-induced reinstatement of drug-seeking. The present study provides a reliable operant model in mice to evaluate a frustrated state following reward unavailability. This animal model could be useful to study the behavioural and neurochemical consequences related to the emotional states generated during the omission of a highly expected reward.

Key Words: Aggressiveness, cocaine, food, frustration, operant behaviour, progressive ratio. 
Introduction

According to the Diagnostic and Statistical Manual of Mental Disorders Fourth edition, and the proposed draft of the Fifth edition, addiction is defined as a chronically relapsing disorder characterized by a progression from impulsive to compulsive behaviour, and the emergence of a motivational withdrawal syndrome when access to the reward is prevented. Drug addiction is generated by maladaptative patterns of consummatory behaviour, similar to what appears in other pathological conditions, associated with natural rewards. Indeed, clinical studies have shown that food craving in obese patients activates areas of the brain analogous to those stimulated during drug craving in addicts (Pelchat et al. 2004; Wang et al. 2004).

A particular aspect of the addictive process that has received less attention is the consequence of the frustrated state occurring when the reward is not available. Thus, the behavioural alterations leading to addictive disorders can be investigated using instrumental behavioural paradigms that basically involve three types of goal directed events: (a) rewarding events represented by the presence of stimuli which evoke a consummatory reaction; (b) punishing events evoked by noxious stimulation at the termination of a behaviour sequence; (c) frustrating events produced by the absence or delay of a rewarding event in a situation where it had been previously present (Amsel 1958). The two first goal directed events have had a considerable amount of theoretical and experimental attention in previous studies (Lynch et al. 2010), whereas the consequences of frustrating events in addiction-like behaviour have received less attention. In animals, the omission of reinforcement after exposure to consistent reinforcement creates a state of frustration that can be measured in subsequent performance (Mackintosh 1974). Human subjects prevented from reaching expected rewards show affective alterations that include aggressiveness, irritability and anxiety 
(Henna et al. 2008). These negative emotional states following frustration may have an important impact on subsequent consumption in drug addicts or patients suffering from food eating disorders. In rodents, frustration models have been mainly implemented to induce a depressive-like state in rats exposed to the total or partial omission of an expected reward (Gray et al. 1977; Louis et al. 2006). Animal models of frustrated food or drug consumption can be of particular relevance to study the behavioural and neurobiological consequences related to the emotional states generated by these frustrated conditions that often occur in drug addicts and food eating disorder patients. In this study, we have developed an original behavioural model of frustrated expected reward by adapting operant behavioural models recently validated in our laboratory to induce and reinstate drug or food-seeking behaviour in mice (Soria et al. 2008; MartinGarcia et al. 2009; Martin-Garcia et al. 2011), and examined the consequences of this state in aggressiveness, anxiety-like behaviour and the reinstatement of reward seeking behaviour. 
Materials and methods

Animals

Male C57BL/6J mice (Charles River, France), weighing 24-26 g at the beginning of the experiment were used in this study. Mice were housed individually in controlled laboratory conditions with the temperature maintained at $21 \pm 1^{\circ} \mathrm{C}$ and humidity at $55 \pm$ $10 \%$. Mice were tested during the first hours of the dark phase of a reversed light/dark cycle (lights off at $8.00 \mathrm{~h}$ and on at $20.00 \mathrm{~h}$ ). Food and water were available ad libitum in mice used in the cocaine experiment. Mice used for operant behaviour maintained by food were food-deprived (85 \% of the initial weight) and water was available ad libitum. Animal procedures were conducted in strict accordance with the guidelines of the European Communities Directive 86/609/EEC regulating animal research and were approved by the local ethical committee (CEEA-PRBB).

Food self-administration apparatus

Operant responding maintained by food was performed in mouse operant chambers (Model ENV-307A-CT, Med Associates, Georgia, VT, USA) equipped with two retractable levers, one randomly selected as the active lever and the other as the inactive. Pressing on the active lever resulted in a pellet delivery together with a stimulus-light named conditioned stimulus (CS), located above the active lever, while pressing on the inactive lever had no consequences. The chambers were made of aluminium and acrylic, and were housed in sound- and light-attenuated boxes equipped with fans to provide ventilation and white noise. A food dispenser equidistant between the two levers permitted delivery of food pellets when required. 
Cocaine self-administration apparatus

Drug self-administration training and testing occurred in operant chambers (Model ENV-307A-CT, Med Associates, Inc., Georgia, VT, USA) equipped with two holes, one randomly selected as the active hole and the other as the inactive. Pump noise and stimuli lights (cues), one located inside the active hole and the other above it were paired with the delivery of the reinforcer. Chambers were made of aluminium and clear acrylic, had grid floors and were housed in sound- and light-attenuated boxes equipped with fans to provide ventilation and ambient noise. When mice responded on the reinforced hole, the stimulus light went on, and a drug infusion was delivered. Cocaine was infused via a syringe that was mounted on a microinfusion pump (PHM-100A, Med Associates, Inc., Georgia, VT, USA) and connected via Tygon tubing (0.96 mm o.d., Portex Fine Bore Polythene Tubing, Portex Limited, Hythe, Kent, UK) to a single channel liquid swivel (375/25, Instech Laboratories, Plymouth Meeting, PA, USA), and to the mouse intravenous (i.v.) catheter. The swivel was mounted on a counterbalanced arm above the operant chamber.

\section{Surgery}

Mice were anaesthetized with a ketamine/xylazine mixture (20 ml/kg of body weight) and then implanted with indwelling i.v. silastic catheters (Soria et al. 2005). Briefly, a 6 cm length of silastic tubing (0.3 mm inner diameter, $0.6 \mathrm{~mm}$ outer diameter) (Silastic ${ }^{\circledR}$, Dow Corning, Houdeng-Goegnies, Belgium) was fitted to a 22-gauge steel cannula (Semat, Herts, UK) that was bent at a right angle and then embedded in a cement disk (Dentalon Plus, Heraeus Kulzer, Wehrheim, Germany) with an underlying nylon mesh. The catheter tubing was inserted $1.3 \mathrm{~cm}$ into the right jugular vein and anchored with suture. The remaining tubing ran subcutaneously to the cannula, which exited at the 
midscapular region. All incisions were sutured and coated with antibiotic ointment (Bactroban, GlaxoSmithKline, Madrid, Spain). After surgery, animals were allowed to recover for 3 days prior to initiation of self-administration sessions. The catheter was flushed daily with heparinised saline (30 USP units/ml). The patency of intravenous catheters was evaluated after the last self-administration session and whenever the behaviour appeared to deviate dramatically from that observed previously by infusion of $0.1 \mathrm{ml}$ thiopental sodium $(5 \mathrm{mg} / \mathrm{ml})$ through the catheter. If prominent signs of anaesthesia were not apparent within $3 \mathrm{~s}$ of the infusion, the mouse was removed from the experiment. The success rate for maintaining patency of the catheter (mean duration of 12 days) until the end of the cocaine self-administration training was $83 \%$. The verification of the catheter patency was not necessary for the extinction and reinstatement phases since cocaine was not available.

\section{Drugs}

Cocaine hydrochloride was obtained from Ministerio de Sanidad y Consumo (Spain) and dissolved in sterile $0.9 \%$ physiological saline. Ketamine hydrochloride (100 mg/kg) (Imalgène 1000; Rhône Mérieux, Lyon, France) and xylazine hydrochloride (20 mg/kg) (Sigma, Madrid, Spain) were mixed and dissolved in ethanol (5 \%) and distilled water (95 \%). This anaesthetic mixture was administered intraperitoneally in an injection volume of $20 \mathrm{ml} / \mathrm{kg}$ of body weight. Thiopental sodium (5 mg/ml) (Braun Medical S.A, Barcelona, Spain) was dissolved in distilled water and delivered by infusion of $0.1 \mathrm{ml}$ through the i.v. catheter. 
Experimental design

A first group of C57BL/6J male mice $(n=73)$ was trained during $1 \mathrm{~h}$ for 10 consecutive days to lever-press for chocolate-flavoured food-pellets as reward (R), paired with the presentation of a cue-light serving as CS, on a fixed ratio (FR) 1 schedule of reinforcement followed by 10 sessions under FR5. The criteria for acquisition of operant responding were achieved when mice maintained a stable responding with less than $20 \%$ deviation from the mean of the total number of food-pellets earned in three consecutive sessions, with at least $75 \%$ responding on the reinforced lever, and a minimum of 10 reinforcements per session (Soria et al. 2008; Martin-Garcia et al. 2011). After the 20 FR sessions, animals were trained in a progressive ratio (PR) schedule where the response requirement to earn the reinforcer escalated according to the following series: 1-2-3-5-12-18-27-40-60-85-115-150. Animals were only exposed to one session of PR during the whole training period. The maximum duration of the PR session was $4 \mathrm{~h}$ or until mice did not respond on any lever within $1 \mathrm{~h}$. Mice were fooddeprived during the whole experiment at $85 \%$ of their ad libitum initial weight adjusted for growth. In mice that reached the end point response (ratio 150) 3 different experimental conditions were implemented: i) a first group receiving chocolateflavoured pellets and the cue-light ( $\mathrm{CS}+\mathrm{R}+)$, interpreted as the positive control, ii) a second group that only received the presentation of the cue-light (CS+R-), interpreted as the frustrating event, iii) a third group that did not receive chocolate nor the cue-light (CS- R-), interpreted as the despair-like situation. An additional control group that did not receive the reinforcer nor the light during all phases of the experiment was also included to control the possible effects of this long operant training on aggressiveness and anxiety-like behaviour (Fig. 1). The end point selected was lower than the breaking point obtained in previous studies under similar experimental conditions (366.88 \pm 
77.73) in order to allow $100 \%$ of mice reaching this end point 150 . Animals that achieved the end point 150 remained in the training box during 10 additional min without any scheduled consequence when mice responded in the active lever (Fig. 1), and their active and inactive lever-press and rate of responding (lever-presses/min) was recorded. Animals did not receive any additional training in the operant chambers before the extinction sessions. Half of the mice of each experimental group were then immediately exposed to the light-dark box test and subsequently to the resident-intruder test, whereas the remaining mice were exposed to the extinction and cue-induced reinstatement phases of the operant paradigm. The first extinction session occurred $24 \mathrm{~h}$ after the PR session.

A second group of mice $(n=87)$ was trained during $2 \mathrm{~h}$ daily sessions to acquire operant responding maintained by cocaine $(0.5 \mathrm{mg} / \mathrm{kg} /$ infusion, i.v. $)$ paired with a cuelight under FR1 (6 consecutive daily sessions) and FR3 (6 consecutive daily sessions). The criteria for the acquisition of cocaine self-administration were the same than those described for food operant training. After the 12 FR sessions, animals were tested in a PR schedule where the response requirement to earn the reinforcer escalated according to the following series: 1-2-3-5-12-18-27-40. The maximum duration of the PR session was $4 \mathrm{~h}$ or until mice did not respond on any hole within $1 \mathrm{~h}$, and was performed only once. Mice were feed ad libitum during the whole experiment. In mice that reached the end point response (ratio 40), 3 different experimental conditions were implemented: i) a first group receiving cocaine and the cue-light, $\mathrm{CS}+\mathrm{R}+$, ii) a second group that only received the presentation of the cue-light, CS+ R-, iii) a third group that did not receive cocaine nor the cue-light, CS- R-. An additional control group that did not receive the reinforcer nor the light during all phases of the experiment was also included to control the possible effects of this long operant training on aggressiveness and anxiety-like 
behaviour (Fig. 1). The end point selected was lower than the breaking point obtained in previous studies under similar experimental conditions $(57.17 \pm 20.09)$ in order to allow $100 \%$ of mice reaching this end point 40 . Animals that achieved the end point 40 remained in the training box during 10 additional min without any scheduled consequence when mice responded in the active hole (Fig. 1), and their active and inactive nose-poking and rate of responding (nose-pokes/min) was recorded, these mice were then tested such as mice exposed to food operant training. After PR session, the thiopental test was applied and only mice that showed patency of catheter were moved to the extinction phase. The first extinction session occurred $48 \mathrm{~h}$ after the thiopental tests in order to avoid any possible influence of thiopental residual effects.

\section{Light-dark box}

The box consists of a small compartment with black walls and black floor dimly illuminated (25 Lux), connected by a $4 \mathrm{~cm}$ long tunnel to a large compartment with white walls and a white floor intensely lit (500 Lux) (Bura et al. 2010). Straight lines drawn on the floor of both compartments allowed the measurement of locomotion (number of squares crossed). Mice were individually placed in the dark compartment facing the tunnel at the beginning of the 5 min observation session. The number of entries in the distal zone of the lit compartment was analyzed as the most significant parameter to reveal anxiety-like behaviour in our experimental conditions.

\section{Resident-intruder test}

This procedure evaluates aggressive-like behaviour in rodents (Martin et al. 2002). Intruder animals of a similar weight were housed 5 per cage. Each session consisted in placing an intruder mouse into the resident's home cage for a period of $4 \mathrm{~min}$. Residents 
were mice exposed to the three different conditions in the PR paradigm after reaching end point. The latency and frequency for the first attack or menace (e.g. tail-beating) displayed by resident mice were measured.

Extinction and cue-induced reinstatement of operant seeking behaviour During the extinction phase, the experimental conditions were similar to the acquisition sessions except that chocolate-flavoured pellets or cocaine were not available and the cue-light was not presented after responding in the active manipulandum. Mice were given $1 \mathrm{~h}$ daily sessions for food operant behaviour extinction and $2 \mathrm{~h}$ daily sessions for cocaine self-administration extinction, 7 days per week during 50 days until the criteria for extinction was achieved: during 3 consecutive sessions mice responded on the active lever $30 \%$ less than the 3 last acquisition days, and made less than 15 active responses per session. After extinction, mice were tested for reinstatement.

Cue-induced reinstatement was conducted under the same conditions used in the FR1 phase except that food or cocaine were not available. Each response on the active manipulandum in this phase led to the presentation of the cue-light for 2 s. The reinstatement criterion were achieved when responding in the active lever or nose-poke doubled with respect to extinction responding.

Statistical analysis

Analysis of the data obtained during the acquisition phase was conducted using threeway ANOVA of repeated measures with day and manipulandum (active/inactive) as within-subjects factors and group as between-subjects factor. Post-hoc analysis (Newman-Keuls) was performed when required. 
Operant responses in the active manipulandum during the 10 min after the frustrating procedure were analyzed using two-way ANOVA with time as within-subject factor and group as between-subject factor. Post-hoc analysis (Newman-Keuls) was performed when required.

To evaluate the extinction and cue-induced reinstatement of food and cocaine-seeking behaviour, three-way ANOVA of repeated measures was performed with experimental phase and manipulandum as within-subjects factors, and group as between-subject factor. Post-hoc analysis (Newman-Keuls) was performed when required. In addition, one-way ANOVA was used to compare the day reaching the extinction criteria of the three different groups.

All results are expressed as mean \pm S.E.M. Differences were considered significant at $p$ $<0.05$. The statistical analysis was performed using the Statistical Package for Social Science program SPSS ${ }^{\circledR} 15.0$ (SPSS Inc, Chicago, USA). 
Results

Operant model of frustrated expected reward after operant training to obtain chocolateflavoured pellets

Acquisition of operant responding maintained by palatable food

The acquisition criteria for operant responding maintained by chocolate-flavoured pellets on FR1 and FR5 were achieved by all the animals equally. No differences in the acquisition or the maintenance of operant responding under FR1 and FR5 schedule were revealed among the three experimental groups that acquired operant responding before the PR training (for three-way ANOVA see table S1A, supplemental information). None of the animals included in the negative control group achieved the acquisition criteria during FR1 or FR5 training, and significant differences were revealed between this negative control and the other experimental groups from the first day of training (Fig. 2A).

\section{Operant responding after the PR}

The operant responding during the 10 additional min after achieving the end point 150 was evaluated without any scheduled consequence when mice responded in the active lever. As expected, none of the animals in the negative control group achieved this end point. Interestingly, the CS+ R- group that underwent the frustrated delivery of the reward paired with the presentation of the CS, showed a significant increase in operant responding after this end point in comparison with the CS+ R+ and the CS- R- groups. Post-hoc Newman-Keuls comparison revealed that the number of active responses in the CS+ R- group was significantly higher 2, 5 and 10 min after the end point compared with CS- R- ( $p<0.01, p<0.01$ and $p<0.05$ respectively), and CS+ R+ groups ( $p<0.05$ in all the time points) (Fig. 3A). In agreement, significantly higher 
operant responding rates (lever-presses/min) were revealed in the CS+ R- group at 2, 5 and 10 min after the end point in comparison with CS- R- $(p<0.01, p<0.01$ and $p<$ 0.05 respectively) and $\mathrm{CS}+\mathrm{R}+$ groups ( $p<0.05$ in all the time points) (Fig. 3B, for oneway ANOVA see table S2A, supplemental information). Response patterns performed on the active and inactive levers during the 10 additional min after the end point for representative mice in each experimental group are depicted in Fig. $3 \mathrm{C}$.

\section{$\underline{\text { Resident-intruder test }}$}

One-way ANOVA for the number of aggressive events revealed a significant effect of group $(F(3,36)=3.824, p<0.05)$. Subsequent Newman-Keuls analysis showed a significant enhancement in the number of aggressive events in the CS+ R- group compared to CS+ $\mathrm{R}+$ and negative control groups $(p<0.01)$ (Fig. 4A). Moreover, oneway ANOVA revealed a significant difference in the latency for the first aggressive behaviour $(F(3,36)=3.229, p<0.05)$. Subsequent Newman-Keuls analysis showed that

the CS+ R- group had significantly reduced latency compared to $\mathrm{CS}+\mathrm{R}+(p<0.05)$ and negative control groups ( $p<0.01)$ (Fig. 4B).

\section{$\underline{\text { Light-dark box }}$}

No differences between groups were observed in the number of entries in the distal area of the lit compartment after the PR end point $(F(3,31)=0.451$, n.s.) (Fig. S1A, supplemental information).

\section{$\underline{\text { Extinction and cue-induced reinstatement }}$}

The extinction criteria were achieved by all the mice after an average of $9.2 \pm 1.81$ sessions of extinction for the CS $+\mathrm{R}+$ group and $10.8 \pm 0.88$ for the CS- R- group, 
whereas the CS+ R- group achieved the criteria later (15.5 \pm 2.40 sessions) (Fig. 5A). However, one-way ANOVA did not show a significant effect of group $(F(2,27)=3.28$, n.s.).

During the first extinction session, the number of inactive responses were higher than during the acquisition phase in the three experimental groups (for three-way ANOVA see table S3A, supplemental information). In this first session, the CS+ R- group showed higher number of active responses than the CS- R- group $(p<0.05)$. After extinction, exposure to the associated cue reinstated food-seeking behaviour in all the animals equally (100 \%). Post-hoc Newman-Keuls comparison revealed that the number of active lever-presses when reaching the extinction criteria was significantly lower than the responses during the acquisition of the FR1 self-administration criteria within each group ( $p<0.001$ ). During the reinstatement test, the number of active responses increased with respect to extinction values in all groups $(p<0.001)$. In contrast, no significant changes in the number of inactive lever-presses were found when comparing the acquisition, extinction and reinstatement phases. In addition, no significant differences between the three experimental groups were revealed in cue-reinstated seeking behaviour (Fig. 5B). The negative control group was not included in extinction and reinstatement phases of the experiment since these mice never achieved the acquisition criteria.

Operant model of frustrated expected reward after operant training to obtain cocaine Acquisition of operant responding maintained by cocaine

The acquisition criteria for operant responding maintained by cocaine (0.5 $\mathrm{mg} / \mathrm{kg} /$ infusion) were achieved by all the animals on FR1 schedule of reinforcement and by $83 \%$ of the animals on FR3. The remaining $17 \%$ of the animals lost the patency of 
the catheter before achieving the acquisition criteria. No differences in the acquisition or the maintenance of operant responding under FR1 and FR3 schedule were revealed among the three experimental groups before the PR training (for three-way ANOVA see table S1B, supplemental information). None of the animals included in the negative control group achieved the acquisition criteria during FR1 or FR3 training, and significant differences were revealed between this negative control and the other experimental groups from the first day of training (Fig. 2B).

\section{Operant responding after the PR}

Operant responding was evaluated during 10 additional min after achieving the end point 40 without any scheduled consequence when mice responded to the active hole. As expected, none of the animals in the negative control group achieved this end point. Interestingly, the CS+ R- group showed a significant increase in operant responding after this end point in comparison with CS+ R+ and CS- R- groups. Post-hoc NewmanKeuls comparison revealed that the number of active responses in the CS+ R- group was significantly higher after the frustrated expected reward compared with the CS+ R+ $(2 \min =p<0.001,5 \min =p<0.01$ and $10 \min =p<0.05)$ and CS- R- groups $(2 \min$ $=p<0.05,10 \mathrm{~min}=p<0.01)$ (Fig. 6A). In agreement, significantly higher operant responding rates (nose-pokes/min) were revealed after the frustrated expected reward in the $\mathrm{CS}+\mathrm{R}$ - group in comparison with $\mathrm{CS}+\mathrm{R}+(2 \mathrm{~min}=p<0.01,5 \mathrm{~min}=p<0.01,10$ $\min =p<0.05)$ and CS- R- groups $(2 \min =p<0.05,10 \mathrm{~min}=p<0.01)$ (Fig. 6B, for one-way ANOVA see table S2B, supplemental information). Response patterns performed on the active and inactive holes during the 10 additional min after the end point for representative mice in each experimental group are depicted in Fig. 6 C. 


\section{$\underline{\text { Resident-intruder test }}$}

One-way ANOVA for the number of aggressive events revealed a significant effect of group $(F(3,23)=5.92, p<0.01)$. Subsequent Newman-Keuls analysis showed a significant enhancement in the number of aggressive events in the CS+ R- group compared to CS- R-, CS+ R+ and negative control groups $(p<0.01)$ (Fig. 7A). Moreover, one-way ANOVA revealed significant differences in the latency of the first aggressive behaviour $(F(3,23)=6.37, p<0.01)$. Subsequent Newman-Keuls analysis showed that the CS+ R- group had significantly reduced latency compared to the CS- R, $\mathrm{CS}+\mathrm{R}+$ and negative control groups $(p<0.01)$ (Fig. 7B).

\section{Light-dark box}

No differences between groups were observed in the number of entries in the distal area of the lit compartment after the progressive ratio end point $(F(3,36)=2.19$, n.s.) (Fig. S1B, supplemental information).

\section{$\underline{\text { Extinction and cue-induced reinstatement }}$}

The extinction criteria were achieved by $94 \%$ of mice after an average of $12.29 \pm 0.99$ sessions for the CS+ R+ group and $14 \pm 1.69$ for the CS- R- group, whereas the CS+ Rgroup achieved the criteria later (15.82 \pm 1.14 sessions) (Fig. 8A). However, one-way ANOVA did not show a significant effect of group $(F(2,57)=2.13$, n.s. $)$.

During the first extinction session, the number of active and inactive responses were higher than during the acquisition phase in the three experimental groups (for three-way ANOVA see table S3A, supplemental information). In this first session, the CS+ Rgroup showed higher number of active responses than the CS- R- group $(p<0.05)$. After extinction, exposure to the associated cue reinstated cocaine-seeking behaviour in 
all the animals. Post-hoc Newman-Keuls comparison revealed that the number of active nose-pokes when reaching the extinction criteria were significantly lower than the responses during the acquisition of the FR1 self-administration criteria within each group ( $p<0.001$ ). During the reinstatement test, the number of active responses increased with respect to extinction values $(p<0.001)$. Interestingly, post-hoc NewmanKeuls analysis revealed a higher number of responses during the reinstatement phase in the CS+ R- group than the CS+ R+ and the CS- R- groups $(p<0.05)$ (Fig. 8B). The negative control group was not included in extinction and relapse phases since these mice never achieved the acquisition criteria. 


\section{Discussion}

In the present study, we have validated for the first time an original operant model in mice to evaluate the behavioural consequences produced by the unexpected absence of a highly desired reward. The rewarding stimulus omitted in this operant paradigm was either palatable food or cocaine. Only the frustrated expectation of reward produced perseverative operant responding and increased aggressiveness. In addition, cue-induced reinstatement was enhanced in the CS+ R- group when the rewarding stimulus omitted was cocaine. The frustration that occurred was specially driven by the cue-light used as CS. In agreement, the group CS- R- exposed to a despair-like situation, not receiving the cue-light, did not show perseverative operant responding and increased aggressiveness in our experimental paradigm.

In order to promote specific behavioural consequences after the frustrated event, we have maximized the effort requested to obtain the reward by using an adapted PR schedule of reinforcement where the animals reached an elevated end point. In this schedule, the number of responses required to receive a reward is increased progressively according to pre-establish scheduled progression, and the maximal amount of work that the animal has to perform before cessation of responding (breaking point) is considered as a reliable index of the motivation for the reward. In our experimental design, we omitted the reward at a selected end point response ratio in order to produce frustration. The points were selected considering the maximum effort that the majority of the mice currently perform in this paradigm in order to obtain each specific reward. Based on the performance of mice in preliminary experiments carried out in our laboratory (see Material and methods section), we chose the $12^{\text {th }}$ point of the sequence, end point 150 , when the rewarding stimulus was palatable food, and the $8^{\text {th }}$ point of the sequence, end point 40 , when it was cocaine. After performing this elevated 
number of active responding, the reward was unexpectedly absent. In addition, we have increased the control of the CS over operant behaviour using partial reinforcement schedules (FR3 or FR5) before the PR session, to consolidate the operant training after acquisition with a continuous reinforcement schedule (FR1). This partial reinforcement has been widely reported to increase perseveration and resistance to extinction in both humans (Nation \& Woods et al. 1980; Eaglen \& Mackenzie 1981) and animals (MartinGarcia et al. 2011), and ensures the high value and expectancy of the reward associated with the CS in our experimental conditions. Under these conditions, only the group CS+ R- developed perseverative response. Importantly, the reward delivery omission introduced in our adapted PR schedule was performed in the presence of the CS. Thus, frustration is produced under the exact situation that has been previously resulted in reward delivery. These particular experimental characteristics provide construct and face validity to the behavioural model. Therefore, the frustration that occurred in this adapted PR situation was specially driven by the cue-light used as CS, and the anticipatory reactions during frustration were the result of a high conditioning to this particular stimulus. In contrast, partial reinforcement could have some minor frustrated consequences due to the lack of the expected reward, but only at early stages of training (Amsel 1958). In the same line, the responses not reinforced during the PR schedule do not have relevant frustrating consequences since they were not signalled with the cuelight. In agreement, the group CS- R- exposed to a despair-like situation, not receiving neither the cue-light nor the reward, did not show the frustrating responses revealed in our experimental paradigm. It is important to underline that the sequence of PR was also being increased across this session and was being reinforced in this CS- R- group, with the exception of the last point. This result confirms the crucial relevance of the cue-light 
in order to promote the behavioural consequences associated to the frustrated expected reward.

In our experimental model, an increase in the number of operant responses and rate of responding was observed immediately after the frustrating event. This behavioural consequence was only revealed in the CS+ R- and was similarly observed when the omitted stimulus was either a natural reward (palatable food) or a drug (cocaine). Both kinds of rewarding stimuli activate the same brain circuits to produce the reinforcing effects and can potentially promote a loss of control over repeated consumption (Volkow, Fowler \& Wang 2003). This result demonstrates a facilitation of persistence of responding immediately after the unexpected absence of reward, which has been postulated to be a main consequence of the frustration reaction (Amsel 1958; Amsel et al. 1964). The burst pattern in operant responding generated by our frustrating event could reflect a negative emotional state and an early symptom of the later development of the compulsive behaviour characteristic of addiction (Belin et al. 2009). Addictive behaviour involves a disruption in cognitive flexibility, which consists in the inability to stop responding to stimuli that have previously predicted the availability of reward or punishment (Stalnaker et al. 2009). This incapability to cope with a new situation might underlie perseverating and compulsive behaviour (Kelley 2004). In this sense, the CS+ R- group in our paradigm reflects difficulties in adapting the behaviour to a new situation of omission of the reward, which could represent an early manifestation of the inflexibility that is hypothesized to characterize addictive behaviour. Mice exposed to the frustrating event also showed enhanced aggressive behaviour in the resident-intruder test. Aggressiveness-related feelings are a well-known consequence of frustration in humans (Henna et al. 2008; Nummenmaa \& Niemi 2004). Enhanced aggressiveness has also been reported in several animal species after operant 
reward extinction (Gray et al. 1977; Louis et al. 2006; Azrin, Hutchinson \& Hake 1966; Dantzer, Arnone \& Mormede 1980; De Almeida \& Miczek 2002). However, this enhanced aggressiveness was not found in animals that were not exposed to the CS after operant responding. The behavioural consequences of the frustrated expected reward are minimized without the exposure to such a CS, as revealed by the absence of significant behavioural manifestations in the CS- R-group exposed to extinction-like conditions in our paradigm. In addition, the maximized effort requested to the mice before the unexpected reward delivery, together with the presence of the CS, ensures the high value and expectancy of the reward, providing the optimal conditions to reveal specific behavioural consequences of the frustrated event. Under these novel experimental conditions, we achieve to differentiate the behavioural consequences of the frustrated expected reward from the effects produced by an extinction-like procedure that is mimicked in our CS- R- group. We did not obtain significant increase of anxiety levels after the frustrated event despite having selected for these experiments the light-dark box test, as the most sensitive paradigm for detecting anxiety-like behaviour in mice in our experimental conditions (Belzung \& Le Pape, 1994; Bura et al. 2010). The lightdark box and other anxiety paradigms are mostly used to detect the anxiolytic or anxiogenic effects produced by drugs (Bourin \& Hascoet 2003). In animals, the sensitivity of these paradigms may not be high enough to detect the subtle changes produced in humans by an event like frustration that are revealed in anxiety tests based on the subjective feelings that humans verbalize, which are much more sensitive than any animal model of anxiety (Henna et al. 2008).

The facilitation of cue-induced reinstatement was revealed in our paradigm only when the omitted stimulus was a drug and not after the absence of the natural reward. The negative consequence of the frustration in cocaine-seeking behaviour points out 
differential long-term behavioural effects promoted by the unexpected absence of a drug. This differential response could be related to the different capability of drugs of abuse and natural rewards to activate the reward circuits and to produce subsequent adaptive changes. The use of the intravenous route of administration would also contribute to these differential consequences after the omission of cocaine delivery (Volkow \& Wise 2005; Di Chiara et al. 2004). These results suggest that the unexpected absence of a drug of abuse could facilitate loss of control over drug-seeking in a higher degree than after the omission of a natural reward under the present experimental conditions. The long-term effect of the single frustrated event represents an interesting and unexpected result that shows the complexity of the behavioural responses evaluated in our study. This long-term effect was only revealed after the cocaine self-administration frustrated event, but not after the frustrated event to obtain palatable food nor in the other experimental groups exposed to cocaine selfadministration. This result reveals the different capability of drugs of abuse and natural rewards to induce long-term effects in the reward circuits. Further studies will be needed to reveal the exact mechanisms underlying the different long-term effects of these two rewarding stimuli.

In conclusion, this original operant model reveals that the immediate consequences on seeking behaviour and aggressiveness of a frustrated expected reward are similar after the omission of a natural reward or a drug of abuse. However, this frustrated event has consequences on the subsequent reinstatement of seeking behaviour only when the omitted stimulus was a drug of abuse. This model provides an excellent tool to reveal the behavioural consequences of a frustrated highly expected reward and the neurobiological mechanisms underlying these behavioural responses that could be crucial for the development of addiction. 
Burokas et al.

Acknowledgements

This work was supported by the DG Research of the European Commission (PHECOMP, no. LHSM-CT-2007-037669 and GENADDICT, no. LSHM-CT-200405166), the Spanish 'Instituto de Salud Carlos III' (RTA, no. RD06/001/001), the Spanish 'Ministerio de Ciencia e Innovación' (no. SAF2007-64062), the Spanish ‘Ministerio de Sanidad y Política Social’ (Plan Nacional Sobre Drogas, no. 2009/026), the Catalan Government (SGR2009-00131), the ICREA Foundation (ICREA Academia-2008). A.B. was supported by a FI predoctoral fellowship of the Catalan Government, J. G-C. was supported by a “Juan de la Cierva” post-doctoral fellowship from the Spanish 'Ministerio de Ciencia e Innovación’ and E. M-G. was supported by a “Sara Borrell” post-doctoral fellowship from the Spanish 'Instituto de Salud Carlos III'. Partial support from FEDER funds is also acknowledged. We thank Maria Erta for invaluable technical assistance, Dr. Manish Kumar Saraf for his collaboration and Dr. Patricia Robledo for critical reading and revision of the manuscript.

Authors contribution

A.B., J. G-C. and E. M-G. conducted the behavioural studies and participated in the interpretation and manuscript writing. R.M. participated in the experimental design, the interpretation and manuscript writing and funded the project. 
References

American Psychiatric Association (2000): Diagnostic and Statistical Manual of Mental

Disorders, fourth edition (DSM-IV) and proposed draft revisions (DSM-5). Washington, DC: American Psychiatric Press.

Amsel A (1958): The role of frustrative nonreward in noncontinuous reward situations. Psychol Bull 55: 102-119.

Amsel A, Mackinnon JR, Rashotte ME, Surridge CT (1964): Partial reinforcement (acquisition) effects within subjects. J Exp Anal Behav 7: 135-138.

Azrin NH, Hutchinson RR, Hake DF (1966): Extinction-induced aggression. J Exp Anal Behav 9: 191-204.

Belin D, Balado E, Piazza PV, Deroche-Gamonet V (2009): Pattern of intake and drug craving predict the development of cocaine addiction-like behavior in rats. Biol Psychiatry 65: 863-868.

Belzung C and Le Pape G (1994) Comparison of Different Behavioral Test Situations Used in Psychopharmacology for Measurement of Anxiety. Physiol Behav 56:623-628.

Bourin M, Hascoet M (2003): The mouse light/dark box test. Eur J Pharmacol 463: 5565.

Bura SA, Burokas A, Martín-García E, Maldonado R (2010): Effects of chronic nicotine on food intake and anxiety-like behaviour in $\mathrm{CB}(1)$ knockout mice. Eur Neuropsychopharmacol 20: 369-378.

Dantzer R, Arnone M, Mormede P (1980): Effects of frustration on behaviour and plasma corticosteroid levels in pigs. Physiol Behav 24: 1-4. 
De Almeida RM, Miczek KA (2002): Aggression escalated by social instigation or by discontinuation of reinforcement ("frustration") in mice: inhibition by anpirtoline: a 5HT1B receptor agonist. Neuropsychopharmacology 27: 171-181.

Di Chiara G, Bassareo V, Fenu S, De Luca MA, Spina L, Cadoni C, Acquas E, Carboni E, Valentini V, Lecca D (2004): Dopamine and drug addiction: the nucleus accumbens shell connection. Neuropharmacology 47: 227-241.

Eaglen A and Mackenzie B (1981): Partial Reinforcement and Extinction of Vasomotor Conditioning. A Test of Cognitive and Two-Factor Theories. Pavlov J Biol Sci 16:108117.

Gray JA, Rickwood L, Drewett RF, Dunne E (1977): Gonadal hormones and effects of partial reinforcement on appetitive behaviour in the rat. Physiol Behav 19: 41-45.

Henna E, Zilberman ML, Gentil V, Gorenstein C (2008): Validity of a frustrationinduction procedure. Rev Bras Psiquiatr 30: 47-49.

Kelley AE (2004): Memory and addiction: shared neural circuitry and molecular mechanisms. Neuron 44: 161-179.

Louis C, Cohen C, Depoortere R, Griebel G (2006): Antidepressant-like effects of the corticotropin-releasing factor 1 receptor antagonist, SSR125543, and the vasopressin 1b receptor antagonist, SSR149415, in a DRL-72 s schedule in the rat. Neuropsychopharmacology 31: 2180-2187.

Lynch WJ, Nicholson KL, Dance ME, Morgan RW, Foley PL (2010): Animal models of substance abuse and addiction: implications for science, animal welfare, and society. Comp Med 60: 177-188. 
Mackintosh (1974): The psychology of animal learning. London; New York, Academic Press.

Martin M, Ledent C, Parmentier M, Maldonado R, Valverde O (2002): Involvement of CB1 cannabinoid receptors in emotional behaviour. Psychopharmacology 159: 379387.

Martin-Garcia E, Barbano MF, Galeote L, Maldonado R (2009): New operant model of nicotine-seeking behaviour in mice. Int J Neuropsychopharmacol 12: 343-356.

Martin-Garcia E, Burokas A, Kostrzewa E, Gieryk A, Korostynski M, Ziolkowska B, Przewlocka B, Przewlocki R, Maldonado R (2011): New operant model of reinstatement of food-seeking behavior in mice. Psychopharmacology 215: 49-70.

Nation JR and Woods DJ (1980): Persistence: the Role of Partial Reinforcement in Psychotherapy. J Exp Psychol 109:175-207

Nummenmaa L, Niemi P (2004): Inducing affective states with success-failure manipulations: a meta-analysis. Emotion 4: 207-214

Pelchat ML, Johnson A, Chan R, Valdez J, Ragland JD (2004): Images of desire: foodcraving activation during fMRI. Neuroimage 23: 1486-1493.

Soria G, Barbano MF, Maldonado R, Valverde O (2008): A reliable method to study cue-, priming-, and stress-induced reinstatement of cocaine self-administration in mice. Psychopharmacology 199: 593-603.

Soria G, Mendizábal V, Touriño C, Robledo P, Ledent C, Parmentier M, Maldonado R, Valverde O (2005) Lack of CB1 cannabinoid receptor impairs cocaine selfadministration. Neuropsychopharmacology 30: 1670-1680. 
Burokas et al.

Stalnaker TA, Takahashi Y, Roesch MR, Schoenbaum G (2009): Neural substrates of cognitive inflexibility after chronic cocaine exposure. Neuropharmacology 56: 63-72.

Volkow ND, Fowler JS, Wang GJ (2003): The addicted human brain: insights from imaging studies. Clin Invest 111: 1444-1451.

Volkow ND, Wise RA (2005): How can drug addiction help us understand obesity? Nat Neurosci 8: 555-560.

Wang GJ, Volkow ND, Thanos PK, Fowler JS (2004): Similarity between obesity and drug addiction as assessed by neurofunctional imaging: a concept review. J Addict Dis 23: 39-53. 
Figure legends

Figure 1. Experimental design. Experimental groups included to reveal the behavioural consequences of the unexpected delivery of a natural reward (palatable food) or a drug of abuse (cocaine). CS, Conditioned stimulus; FR, Fixed ratio; PR, Progressive ratio; R, Reward.

Figure 2. Acquisition of operant behaviour to obtain palatable food or cocaine in mice. (A) Mean number of active lever-presses during FR1 and FR5 schedule of reinforcement to obtain chocolate flavoured-pellets in CS+R+ group $(n=21)$, negative control $(n=10)$, CS+R- group $(n=21)$ and CS- R- group $(n=21)$. (B) Mean number of active nose-pokes during FR1 and FR3 schedule of reinforcement to obtain cocaine in CS+R+ group $(n=21)$, negative control group $(n=10)$, CS+ R- group $(n=21)$ and

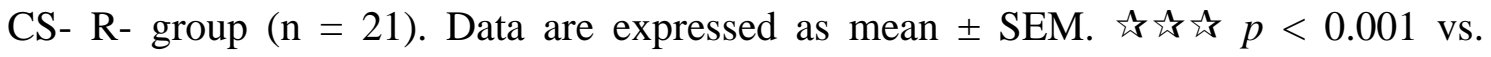
negative control (one-way ANOVA).

Figure 3. Active lever-presses after the frustrated expected reward in mice trained to obtain palatable food. (A) Number of active lever-presses during 10 additional min after end point 150. (B) Active responding rates during the 10 additional min after end point 150. (C) Representative patterns of active and inactive lever-presses during the 10 additional min after the end point 150 for the different groups. Each vertical line represents one active or inactive lever-press. The horizontal line represents the 10 additional min after the end point 150; the upper pattern corresponds to the active and the lower to the inactive lever-presses responses. Data are expressed as mean \pm SEM (n $=21$ in all groups). $\star p<0.05$ vs. CS $+\mathrm{R}+$ group, $\star p<0.05, \star \star p<0.01$ vs. CS- Rgroup (one-way ANOVA). 
Figure 4. Resident-intruder test after the frustrated expected reward (palatable food). (A) Number of aggressive behaviour events. (B) Latency of the first aggressive behaviour. Data are expressed as mean \pm SEM ( $n=10-11$ in all groups). is $p<0.05$, $\star \star s p<0.01$ vs. CS+ R+ group, $\star \star p<0.01$ vs. negative control group (one-way ANOVA).

Figure 5. Extinction of operant responding to obtain palatable food and cue-induced relapse after the frustrated expected reward. (A) Time (days) requested to achieve the extinction criteria. (B) Mean number of lever-presses in the active and the inactive lever during the different experimental phases: mean of the 3 days of the acquisition criteria of food maintained operant behaviour, first extinction session, mean of the 3 days of the acquisition of the extinction criteria and cue-induced reinstatement. Data are expressed

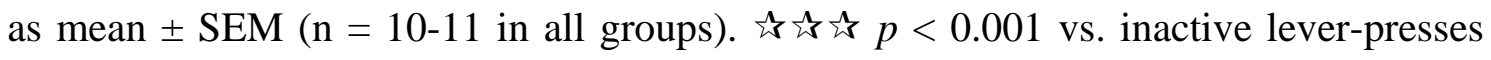
within the same experimental phase; $\star p<0.05, \star \star \mathrm{p}<0.01, \star \star \star p<0.001$ vs. acquisition within the same experimental group; $++p<0.01,{ }^{+++} \mathrm{p}<0.001 \mathrm{vs}$. first extinction session within the same experimental group; \#\#\# $p<0.001$ vs. extinction within the same experimental group; $b p<0.05$ vs. CS- R- group within the same experimental phase (one-way ANOVA).

Figure 6. Active nose-pokes after the frustrated expected reward in mice trained to obtain cocaine. (A) Number of nose-pokes in the active hole during 10 additional min after end point 40. (B) Active responding rates during the 10 additional min after end point 40. (C) Representative patterns of active and inactive nose-poking during the 10 additional min after the end point 40 for the different groups. Each vertical line 
represents one active or inactive nose-poke. The horizontal line represents the 10 additional min after the end point 40; the upper pattern corresponds to the active and the lower to the inactive nose-poking responses. Data are expressed as mean \pm SEM ( $n=21$

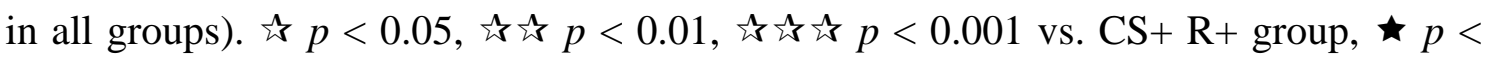
0.05, $\star \star p<0.01$ vs. CS- R- group (one-way ANOVA).

Figure 7. Resident-intruder test after the frustrated expected reward (cocaine). (A) Number of aggressive behaviour events. (B) Latency of the first aggressive behaviour. Data are expressed as mean \pm SEM ( $n=10$ in all groups). is $\dot{2} p<0.01$ vs. CS+ R+ group, $\star \star p<0.01$ vs. negative control group, $b b p<0.01$ vs. CS- R- group (one-way ANOVA).

Figure 8. Extinction of operant responding to obtain cocaine and cue-induced relapse after the frustrated expected reward. (A) Time (days) requested to achieve the extinction criteria. (B) Mean number of nose-pokes in the active and the inactive hole during the different experimental phases: mean of the 3 days of the acquisition criteria acquisition of cocaine maintained operant behaviour, first extinction session, mean of the 3 days of extinction criteria and cue-induced reinstatement. Data are expressed as mean \pm SEM (n

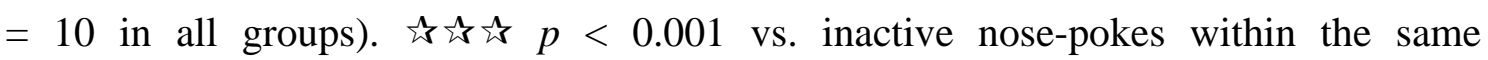
experimental phase; $\star p<0.05, \star \star p<0.01, \star \star \star p<0.001$ vs. acquisition within the same experimental group; $+++p<0.001$ vs. first extinction session within the same group; \#\#\# $p<0.001$ vs. extinction within the same experimental group; $a p<0.05$ vs. $\mathrm{CS}+\mathrm{R}+$ group within the same experimental phase; $b p<0.05$ vs. CS- R- group within the same experimental phase (one-way ANOVA). 


\section{Figure 1.}

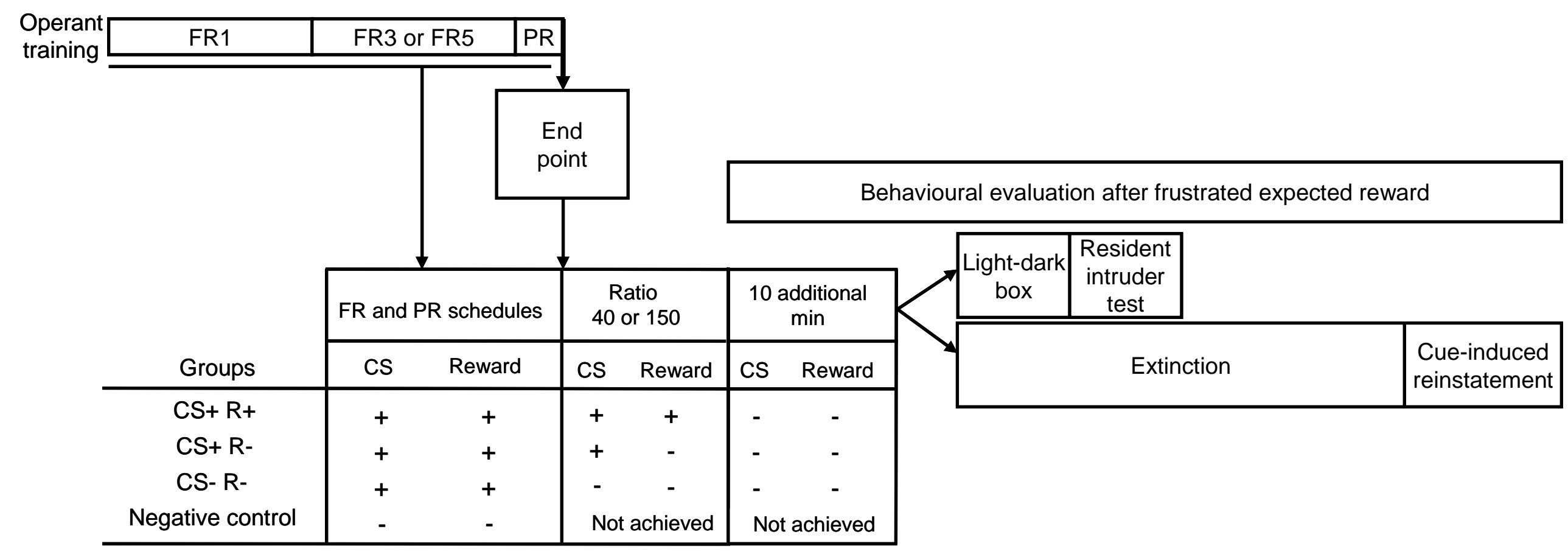


Figure 2.

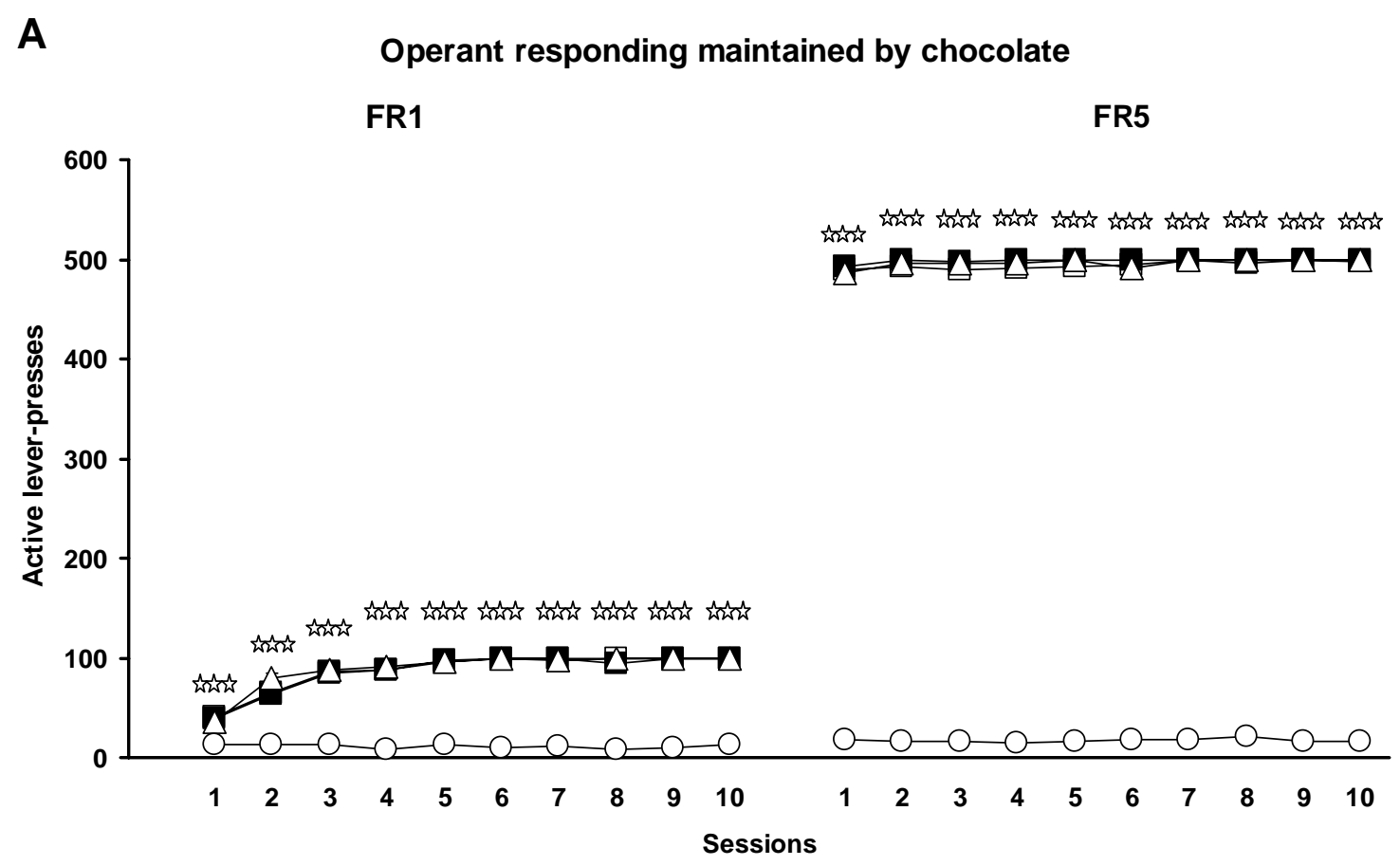

B

Cocaine self-administration

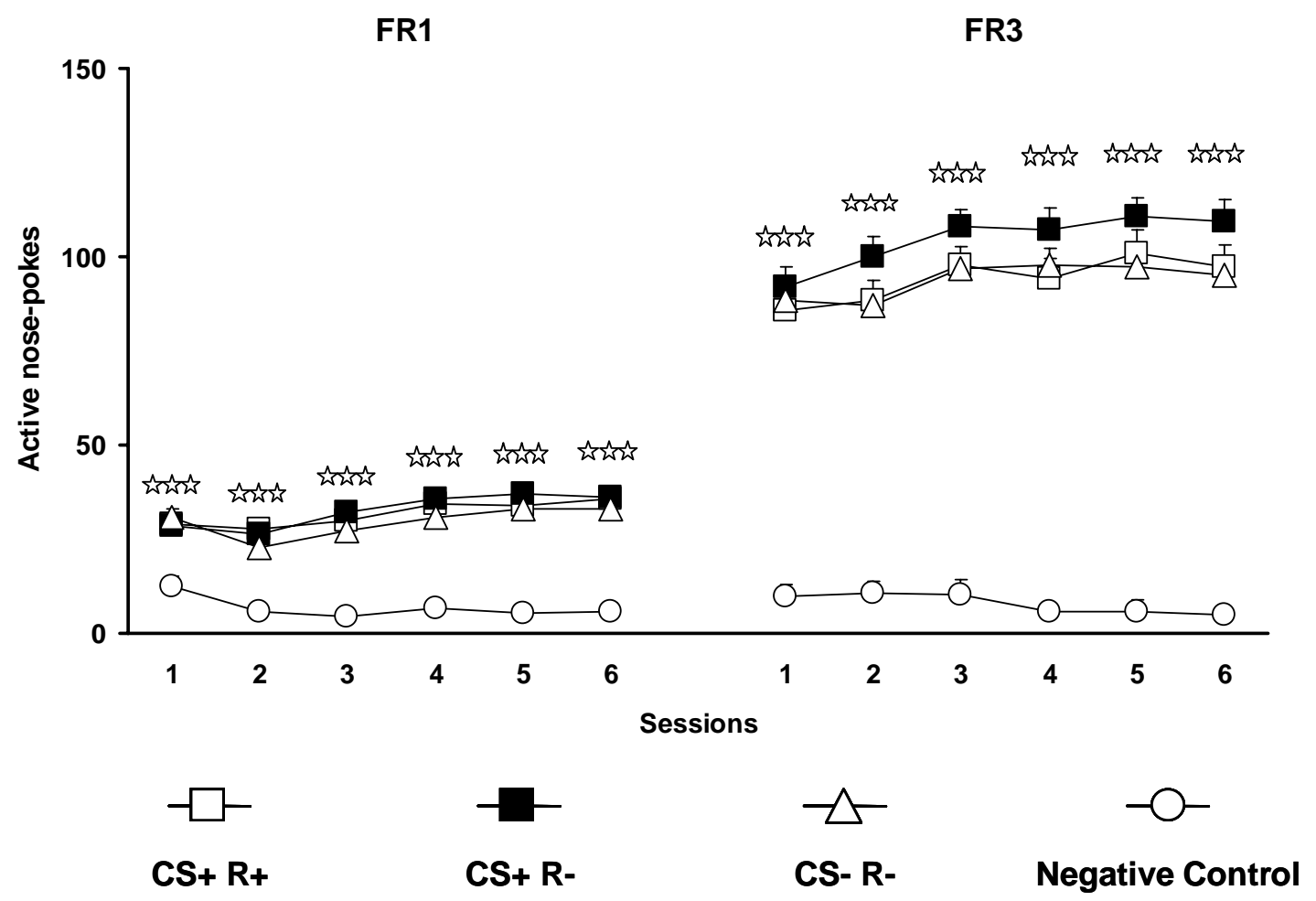


Burokas et al.

Figure 3.

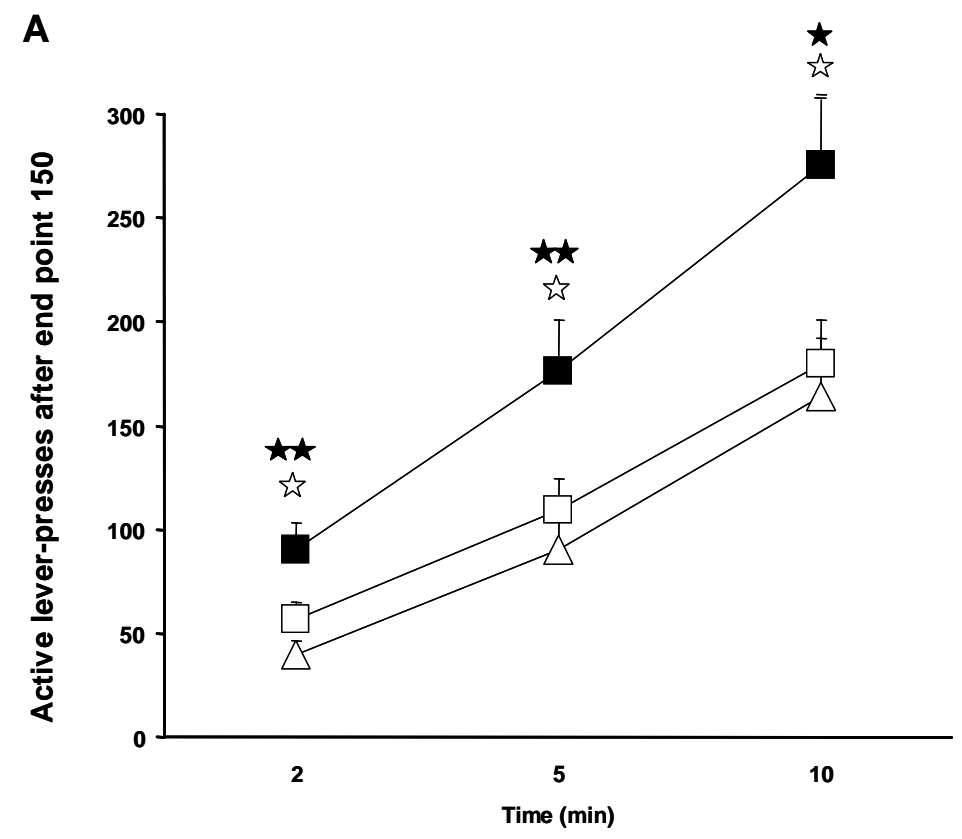

B
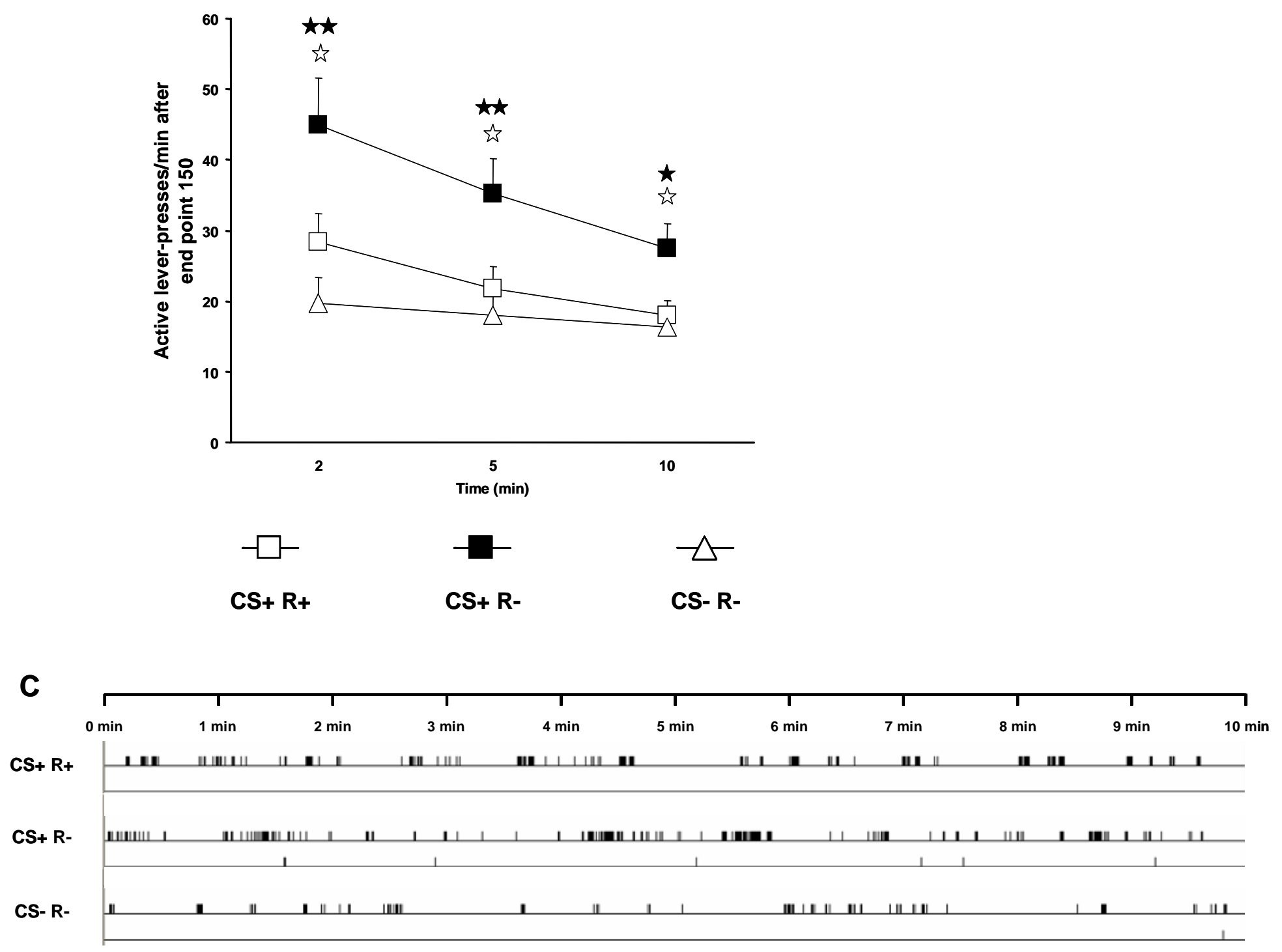
Figure 4.

A

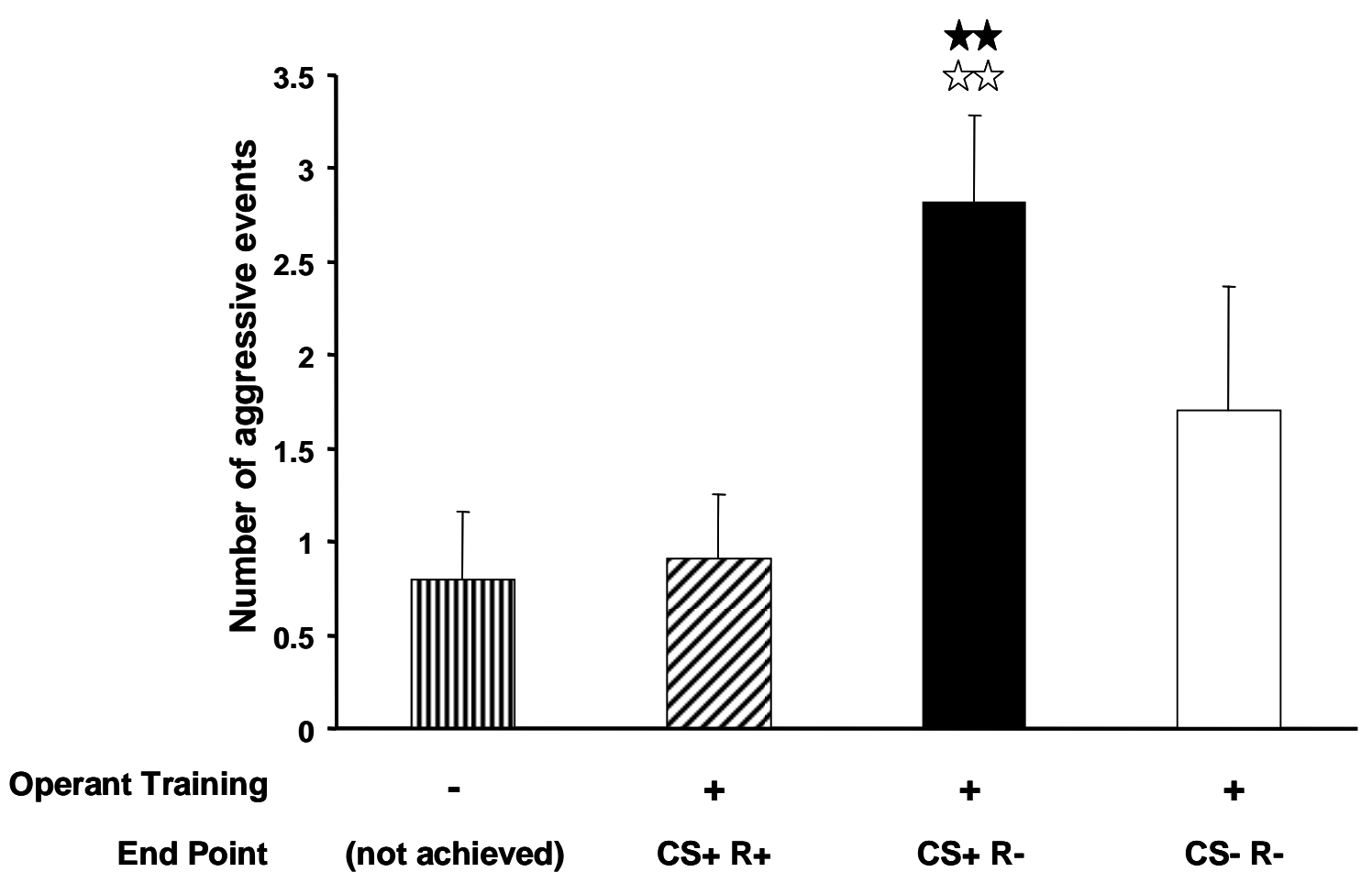

B

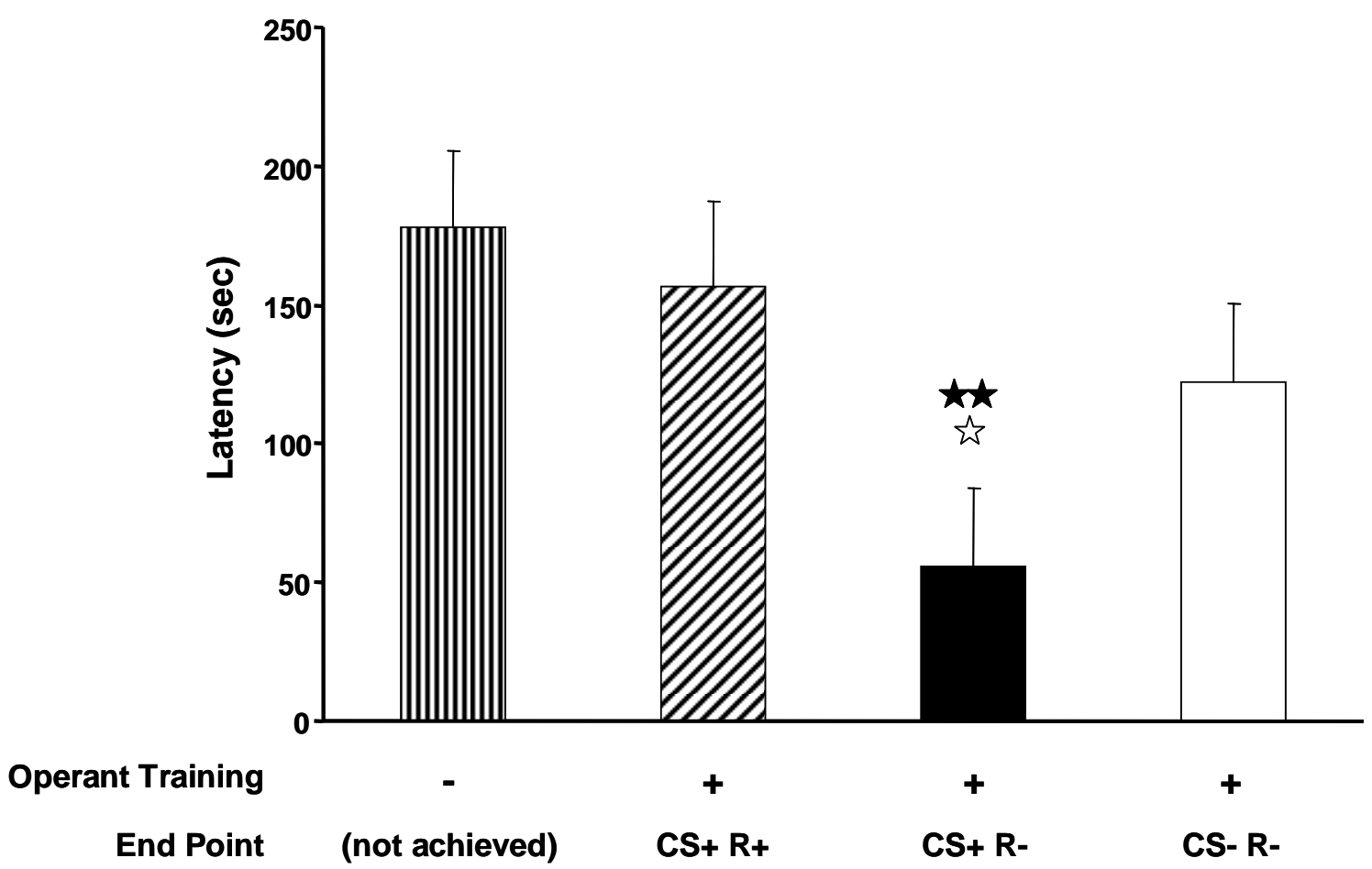


Burokas et al.

Figure 5.

A

Extinction

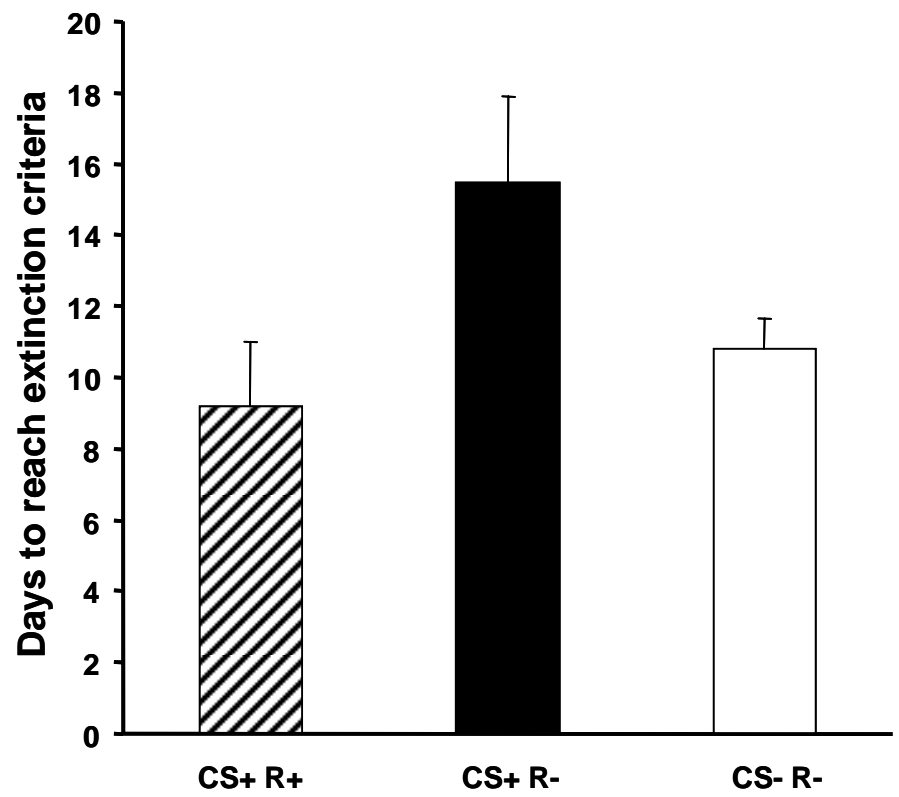

B

active

$\square$ Inactive

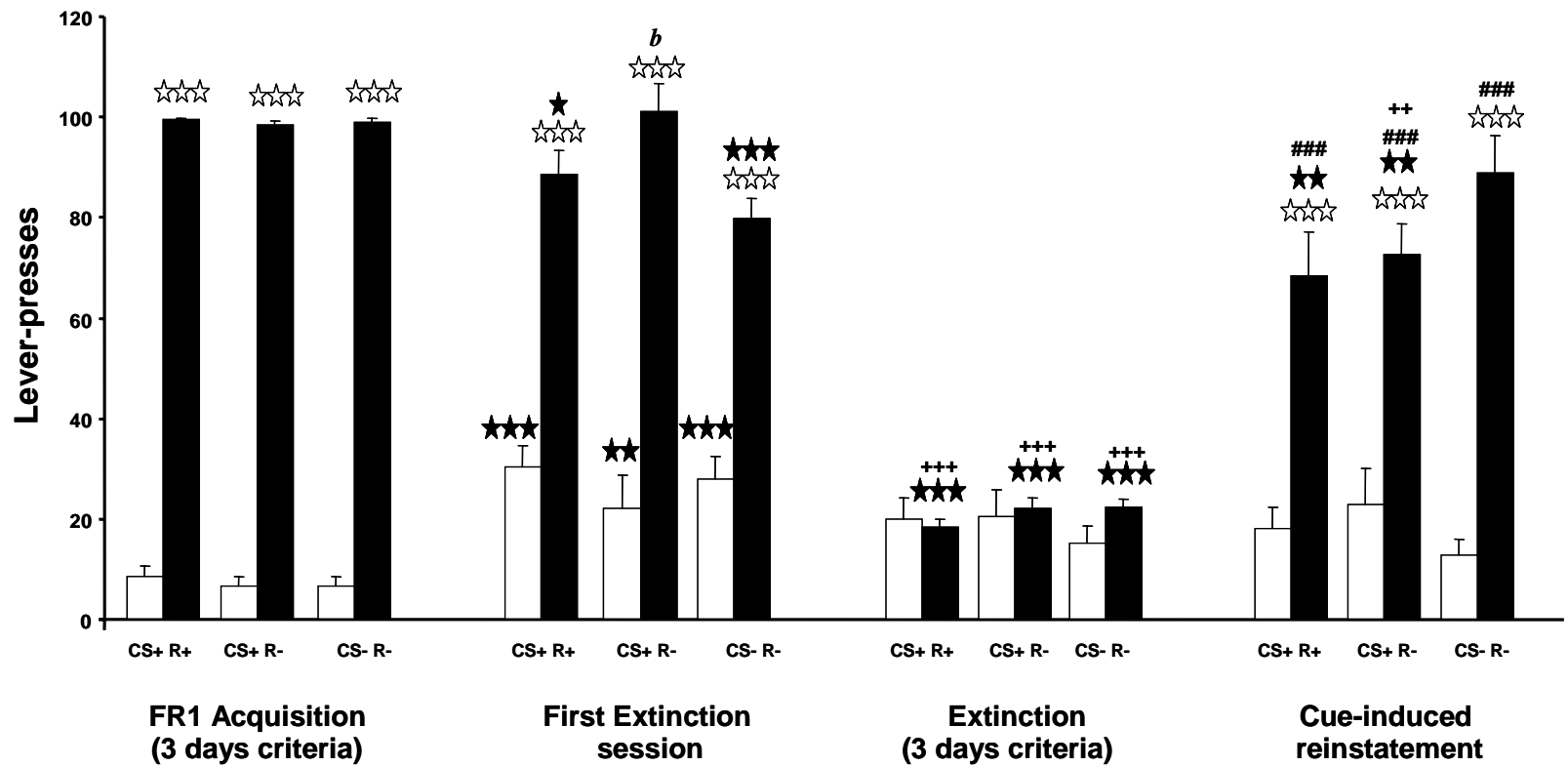


Burokas et al.

Figure 6.

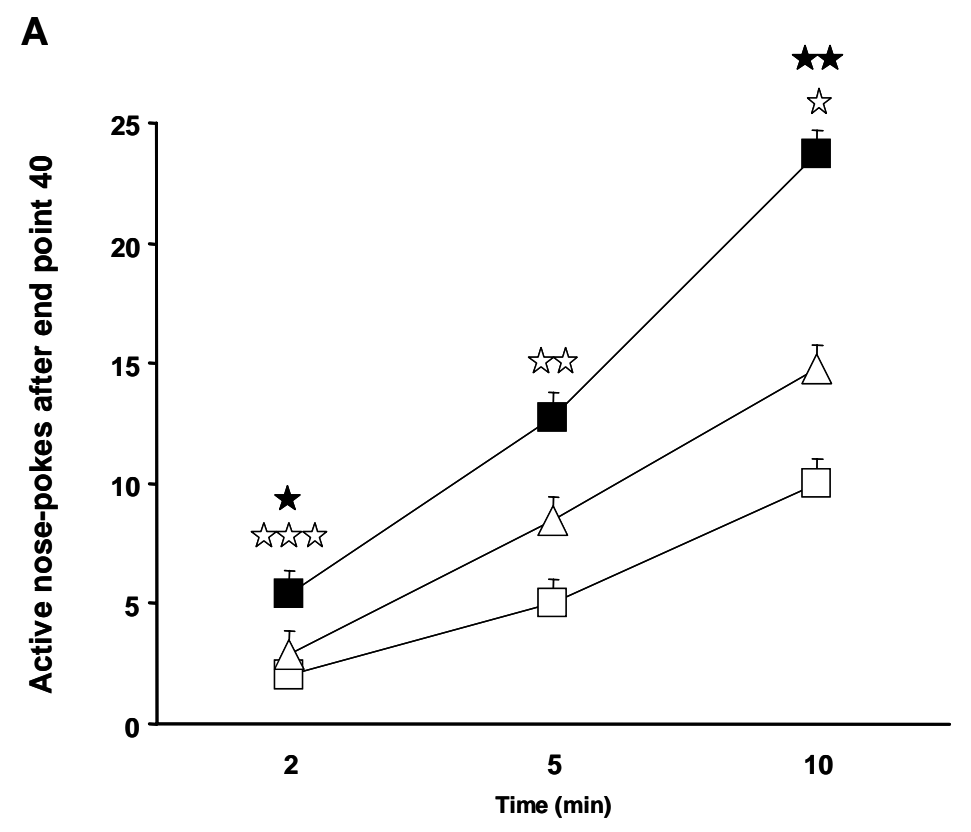

B

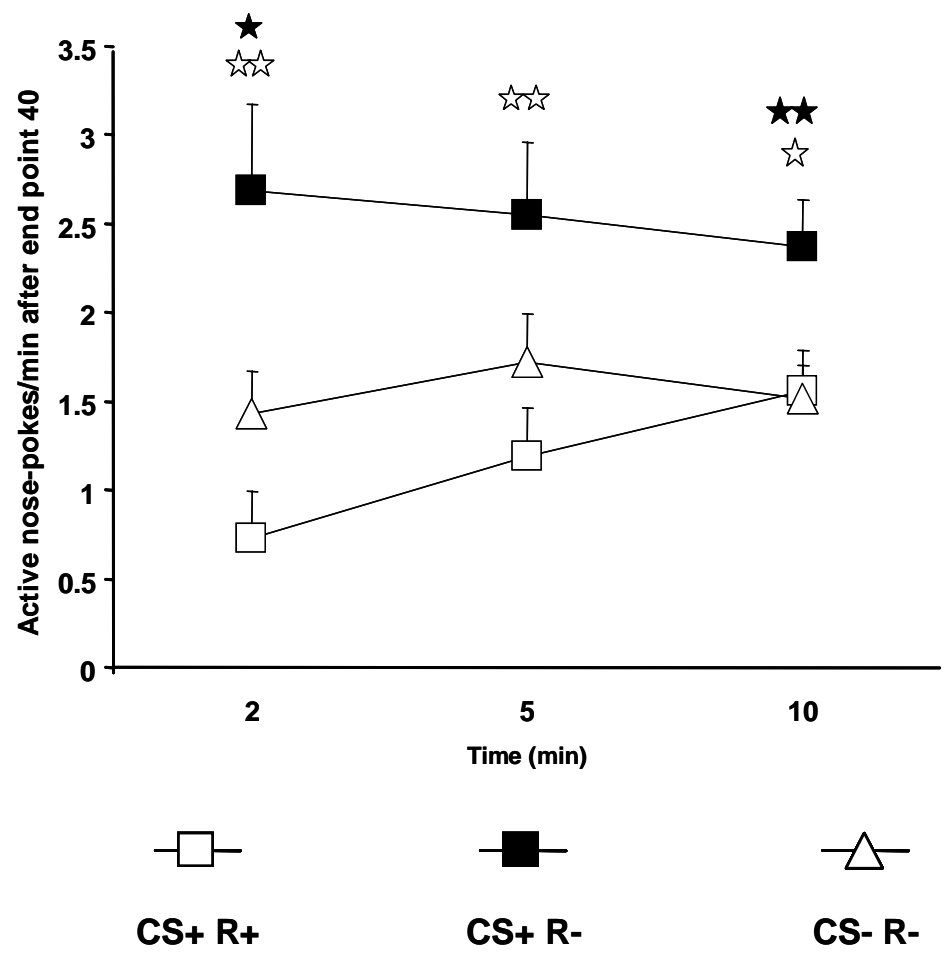

C

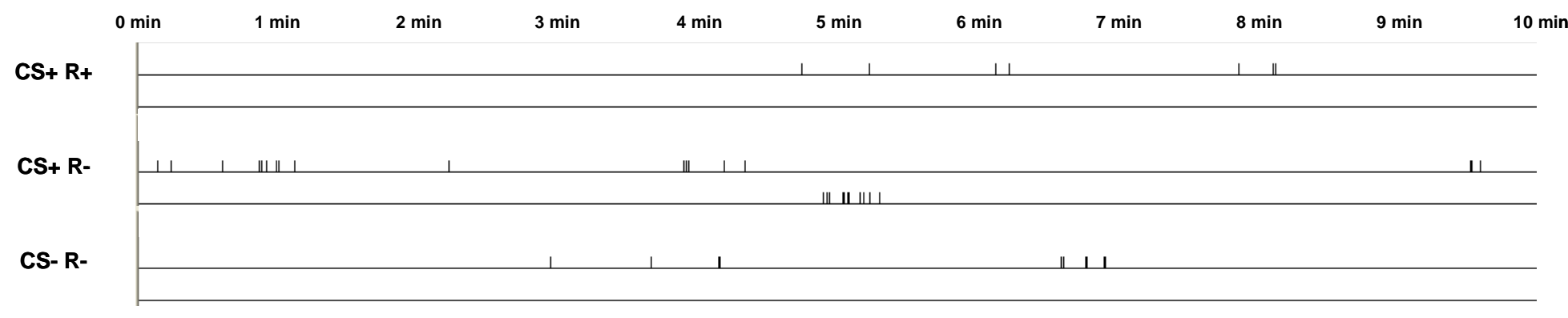


Figure 7.

A

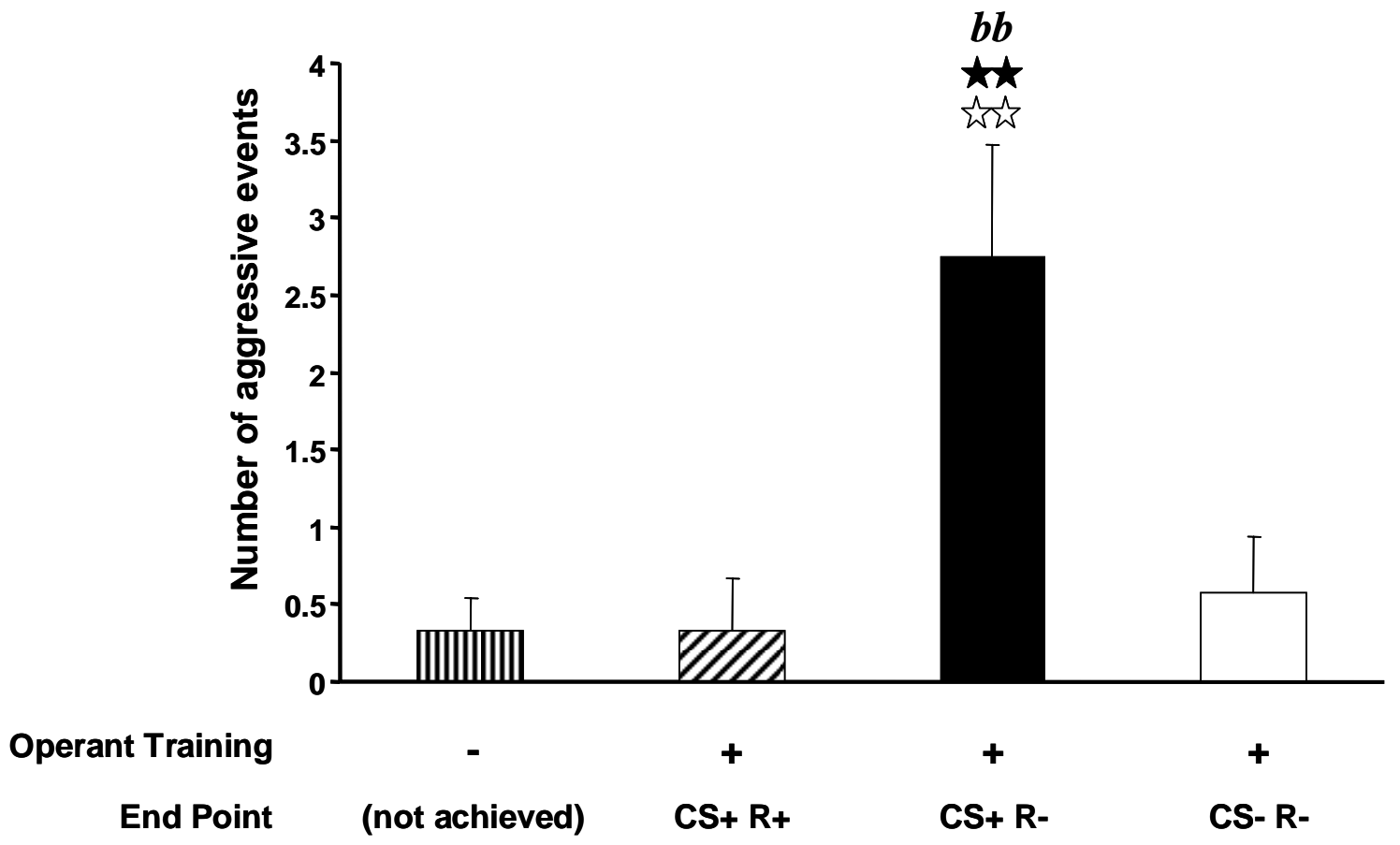

B

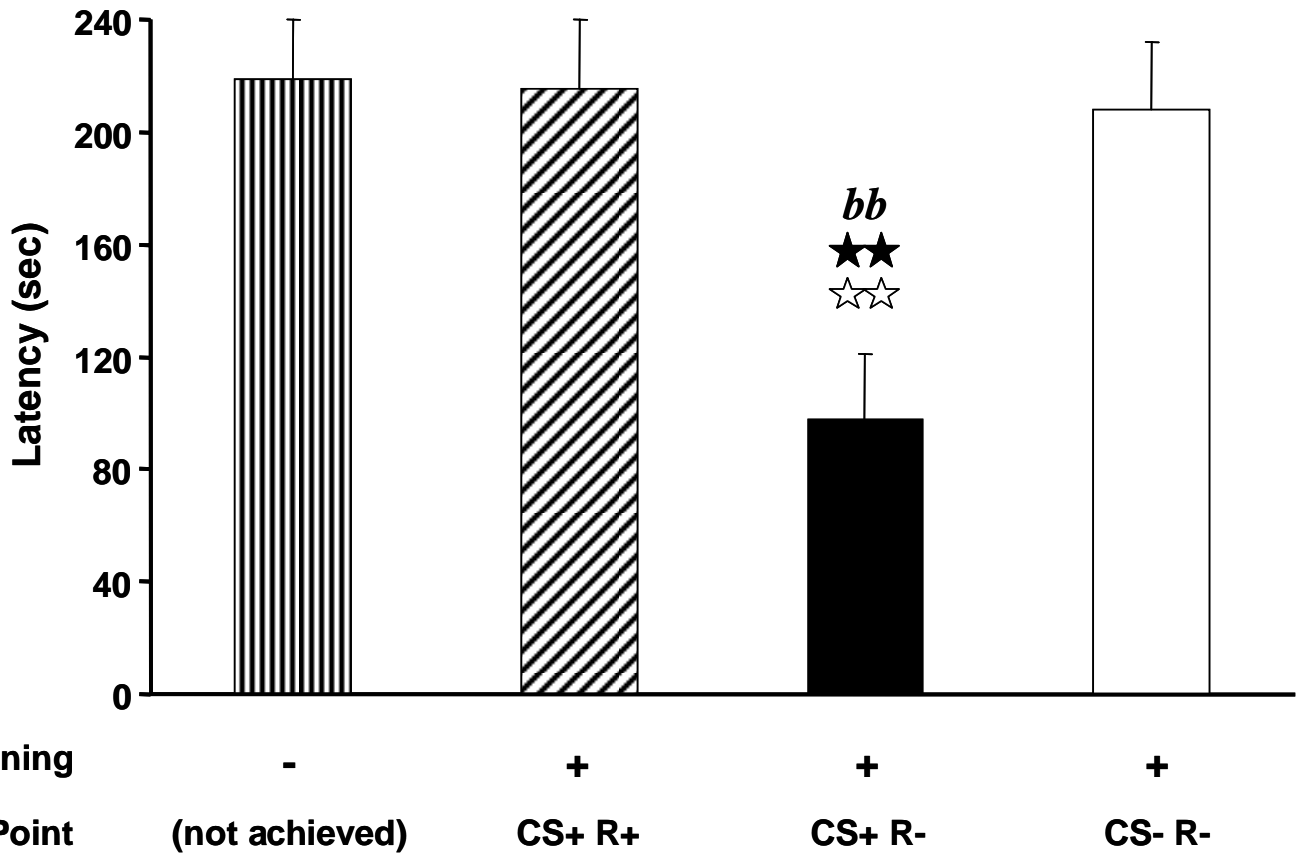


Figure 8.

A

\section{Extinction}

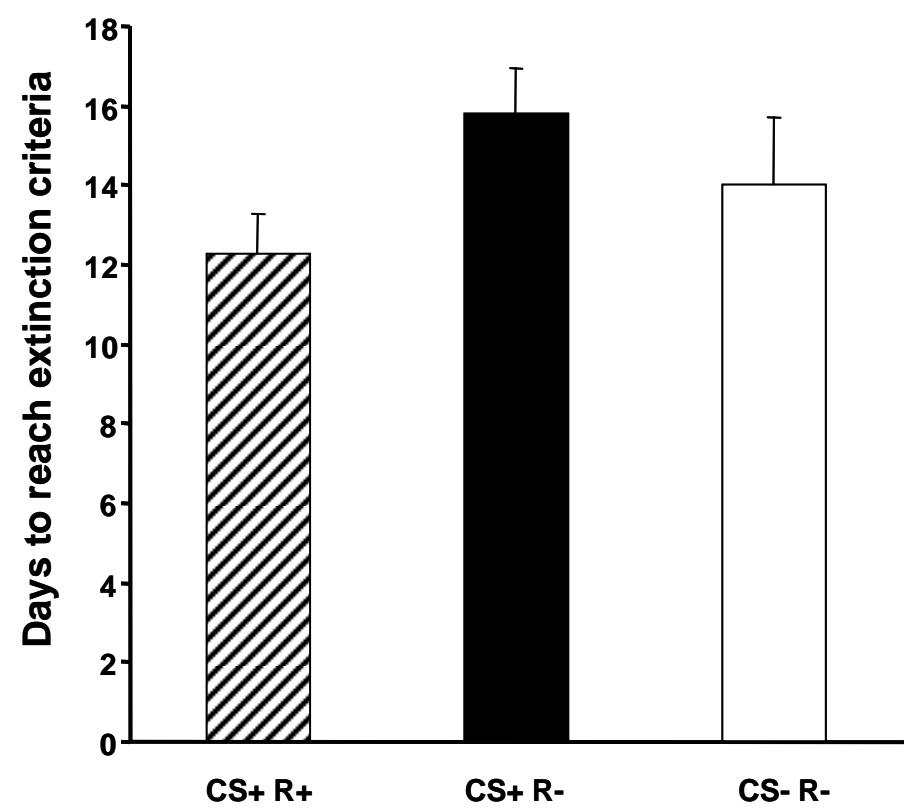

B

\section{Active}

Inactive

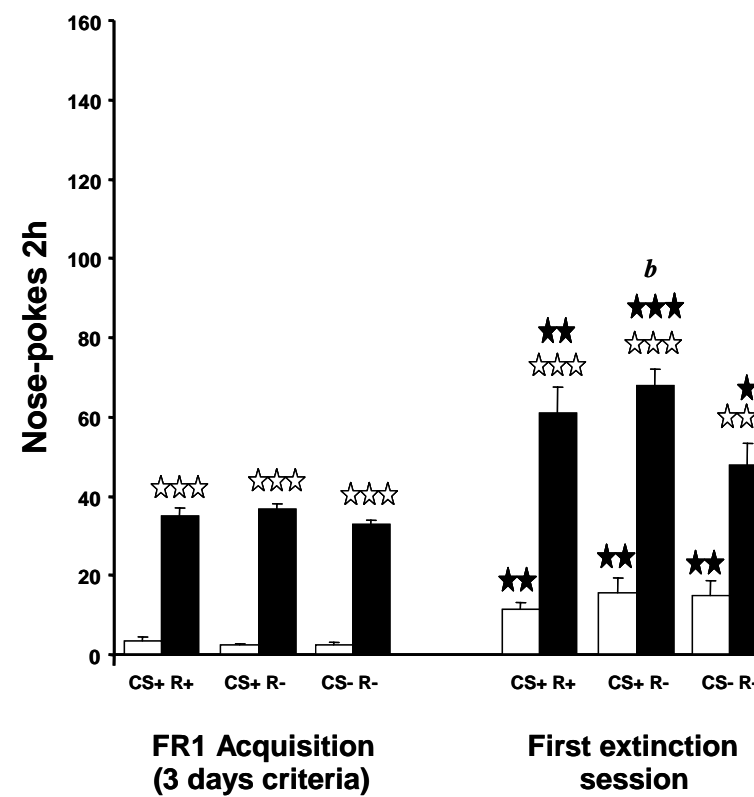

(3 days criteria)

$$
\text { session }
$$

Extinction

(3 days criteria)

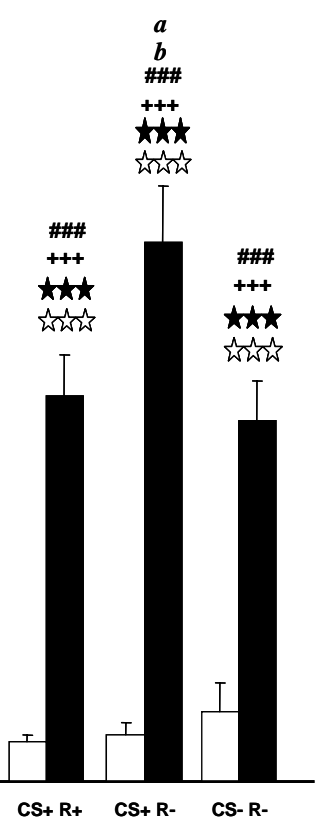

Cue-induced reinstatement 